\title{
Anemia neonatal grave secundaria a transfusión feto-materna: a propósito de un caso
} Severe neonatal anemia due to fetomaternal hemorrhage: an ilustrative case

\author{
Dra. Cristina Menéndez Hernando ${ }^{a}$ Dra. Rocío Chacón Aguilara, Dra. Mercedes Fariñas Salto \\ Dra. Rocío Pérez Crespo ${ }^{a}$, Dra. Raquel Martin Molina ${ }^{a}$, Dra. Rocío Moreno Novillo ${ }^{a}$ \\ Dra. María Cabrerizo Ortiz
}

\begin{abstract}
RESUMEN
La transfusión feto-materna es el paso de eritrocitos fetales a la circulación materna. Cuando es masiva, tiene una incidencia aproximada del $0,2-0,9 \%$. Generalmente, se desconoceel agente desencadenante, pero, en ocasiones, se pueden identificar factores de riesgo. En el embarazo, suele ser asintomática; el síntoma más frecuente es la disminución de los movimientos fetales (el 26\%) en relación con la anemia grave. Se diagnostica mediantela detección dehemoglobina fetal en la sangrematerna (test de Kleihauer o citometría de flujo). Se presenta a un recién nacido con anemia crónica secundaria a la transfusión fetomaterna, que, después del tratamiento con transfusión de concentrado de hematíes, tuvo como complicación síntomas de sobrecarga de volumen y empeoramiento clínico. Tras la realización de una exanguinotransfusión, evolucionó favorablemente, sin secuelas.

Palabras clave: transfusión feto-materna, anemia neonatal, test de Kleihauer-Betke, exanguinotransfusión.
\end{abstract}

\begin{abstract}
Fetomaternal transfusion (FMT) is defined by the transfer of fetal blood into the maternal circulation. The incidence of massive FMT is estimated to be approximately 0.2-0.9 \%o of births. Although a number of etiologies have been associated with FMT, most causes remain unidentified and the pregnancy is usually asymptomatic. The most frequent symptom is the decrease in fetal movements $(26 \%)$ in relation to severe anemia. Several diagnostic modalities for FMT are described (Kleihauer stain, flow cytometry). We describe a case of a newborn with chronic anemia secondary to FMT who, after treatment with transfusions of red blood cells, presented volume overload and clinical worsening as a complication. In this case, our patient needed exchange transfusion for definitive improvement without disability.

Key words: fetomaternal transfusion, neonatal anemia, KleihauerBetke test, exchange transfusion.
\end{abstract}

http: / / dx.doi.org/10.5546/ aap.2019.e142

a. Hospital Universitario Infanta Leonor, Madrid, España.

\author{
Correspondencia: \\ Dra. Cristina Menéndez Hernando: \\ cristinamenendezh@gmail.com
}

Financiamiento: Ninguno.

Conflicto de intereses: Ninguno que declarar.

Recibido: 5-3-2018

Aceptado: 28-11-2018
Cómo citar: Menéndez Hernando C, Chacón Aguilar R, Fariñas Salto M, Pérez Crespo R, et al. Anemia neonatal grave secundaria a transfusión feto-materna: a propósito de un caso. Arch Argent Pediatr 2019;117(2):e142-e146.

\section{INTRODUCCIÓN}

La transfusión feto-materna (TFM) es el paso de eritrocitos fetales a la circulación materna. El intercambio bidireccional es fisiológico y ocurre, principalmente, en el tercer trimestre. ${ }^{1,2}$

La TFM masiva se considera la pérdida absoluta de $80 \mathrm{ml}-150 \mathrm{ml}$ de sangre fetal o relativa de $>20 \mathrm{ml} / \mathrm{kg}$ de la volemia fetal. El diagnóstico de sospecha es fundamental para evitar la elevada morbimortalidad fetal. ${ }^{1-3}$ En el $80 \%$, se desconoce la etiología, y las pruebas confirmatorias (test de Kleihauer, citometría de flujo) no dan resultados inmediatos, ${ }^{1,4,5}$ por lo que el manejo debe realizarse ante la sospecha clínica. Clásicamente, el tratamiento se basa en restaurar la capacidad de transporte de oxígeno $\left(\mathrm{O}_{2}\right)$ y la volemia con transfusión de concentrado de hematíes, aunque, en ocasiones, esa sobrecarga de volumen puede dar lugar a un empeoramiento clínico con resultados fatales. ${ }^{4-8} \mathrm{El}$ tratamiento de esta patología debe realizarse de manera rápida y óptima en función de la situación hemodinámica del paciente.

Se presenta un caso clínico de TFM masiva que precisó exanguinotransfusión para el manejo de la sobrecarga de volumen tras una primera transfusión de concentrado de hematíes.

\section{CASO CLÍNICO}

Primigesta de 18 años, sin antecedentes de interés. Gestación controlada: analíticas, serologías y ecografías normales. Grupo $0, \mathrm{Rh}+$. Acudió con $34^{3}$ semanas por la disminución de los movimientos fetales. Exploración obstétrica sin alteraciones. Registro cardiotocográfico (RCTG): frecuencia normal con desaceleraciones variables aisladas. Se realizó la inducción tras la administración de corticoides. Parto eutócico. Varón, con peso de 2320 g (P52). Precisó 
ventilación manual 2 minutos por hipotonía, bradicardia y marcada palidez. Posteriormente, soporte con presión positiva continua en la vía aérea (continuous positive airway pressure; $C P A P$, por sus siglas en inglés) por dificultad respiratoria. Apgar 6/7/9. Gasometría de cordón: $\mathrm{pH}$ de 7,32; presión parcial de dióxido de carbono $\left(\mathrm{pCO}_{2}\right)$ de $34 \mathrm{mmHg}$; bicarbonato $\left(\mathrm{HCO}_{3}\right)$ : 17,5 mmol/L; láctico: 7,4 mmol/ L; hemoglobina incalculable (Tabla 1).

Al momento de su ingreso a la Unidad de Cuidados Intensivos Neonatales (UCIN), se hallaba taquicárdico, con ligera hipotensión. Presentaba regular estado general, intensa palidez, relleno capilar enlentecido, decaimiento e hipotonía. Fontanela normotensa, sin cefalohematomas, sin edemas ni masas abdominales. Hepatomegalia de
$4 \mathrm{~cm}$ del reborde costal. Grupo sanguíneo $0, \mathrm{Rh}+$ y Coombs directa negativa. Se inició CPAP nasal por la dificultad respiratoria y se mantuvieron saturaciones de oxígeno $\left(\mathrm{SatO}_{2}\right)$ mayores del $94 \%$ en todo momento. A los 40 minutos de vida, presentó equilibrio ácido base venoso (canalización umbilical) con acidosis metabólica importante (elevación de láctico a 11,1 $\mathrm{mmol} / \mathrm{L}$ ); presión parcial de oxígeno $\left(\mathrm{pO}_{2}\right): 29 \mathrm{mmHg}$; hemoglobina: 3,4 g/ dl; hematocrito: el 12,4\%. Desde su ingreso, se inició la expansión con suero fisiológico $(10 \mathrm{ml} / \mathrm{kg})$, se transfundió sangre $0 \mathrm{Rh}-(20 \mathrm{ml} / \mathrm{kg})$ y se administró una dosis de furosemida. Presentó mejoría de la hemoglobina, de 9,5 g/dl, pero persistió la acidosis metabólica sin descenso de láctico y tendencia a la polipnea, bradicardia de 100 latidos por minuto (lpm) y

TABla 1. Parámetros clínicos, valores analíticos y tratamientos realizados

\begin{tabular}{|c|c|c|c|c|}
\hline & Clínica & Constantes & Analítica & Tratamiento \\
\hline $\begin{array}{l}\text { Ingreso en } \\
\text { la UCIN }\end{array}$ & Palidez intensa. & $\begin{array}{l}\text { SatO }{ }_{2} \text { del } 94 \% . \\
\text { TA: } 52 / 31 \mathrm{mmHg} . \\
\text { FC: } 150 \mathrm{lpm} .\end{array}$ & $\begin{array}{c}\text { EAB de sangre de cordón: } \\
\mathrm{pH} \text { de } 7,32 ; \mathrm{pO}_{2}: 28 \mathrm{mmHg} ; \\
\mathrm{pCO}_{2}: 34 \mathrm{mmHg} ; \\
\mathrm{HCO}_{3}: 17,5 \mathrm{mmol} / \mathrm{L} ; \\
\text { láctico: } 7,4 \mathrm{mmol} / \mathrm{L} ; \\
\text { hemoglobina incalculable. }\end{array}$ & CPAP. \\
\hline 40 min de vida & $\begin{array}{c}\text { Palidez, } \\
\text { mala perfusión. } \\
\text { Decaído e hipotónico. }\end{array}$ & $\begin{array}{l}\text { SatO2 del } 94-95 \% . \\
\text { TAM: } 31-35 \mathrm{mmHg} . \\
\text { FC: } 150-160 \mathrm{lpm} .\end{array}$ & $\begin{array}{c}\text { EAB (vena umbilical): } \\
\text { pH de 7,16; } \mathrm{pO}_{2}: 29 \mathrm{mmHg} ; \\
\mathrm{pCO}_{2}: 34 \mathrm{mmHg} ; \mathrm{HCO}: 10 \mathrm{mmol} / \mathrm{L} ; \\
\text { EB: }-16,6 \mathrm{mmol} / \mathrm{L} ; \text { láctico: } 11,1 \mathrm{mmol} / \mathrm{L} \text {. } \\
\text { Hemograma: hemoglobina } \\
\text { de 3,4 g/ dl; hematocrito: el 12,4 \%; } \\
\text { reticulocitos: el } 9 \% ; \\
\text { el resto estaba normal, } \\
\text { incluidas las plaquetas. }\end{array}$ & $\begin{array}{l}\text { Expansión SSF de } \\
10 \mathrm{cc} / \mathrm{kg} \text {. } \\
\text { Transfusión de } \\
\text { concentrado de } \\
\text { hematíes } 0 \mathrm{Rh} \text { - } \\
20 \mathrm{cc} / \mathrm{kg} \text { en } 2 \mathrm{~h} \text {. } \\
\text { Furosemida: } 1 \mathrm{mg} / \mathrm{kg} \\
\text { 1,5 h de vida (antes de } \\
\text { finalizar la transfusión). }\end{array}$ \\
\hline $4 \mathrm{~h}$ de vida & $\begin{array}{c}\text { Tendencia a polipnea } \\
\text { y bradicardia } \\
\text { (FC: } 100 \mathrm{lpm} \text { ) } \\
\text { por sobrecarga } \\
\text { de volumen. }\end{array}$ & $\begin{array}{l}\mathrm{SatO}_{2} \text { del } 98 \% . \\
\text { TAM: } 29-31 \mathrm{mmHg} . \\
\text { FC: } 100 \mathrm{lpm} . \\
\text { hen } \\
\text { re }\end{array}$ & $\begin{array}{c}\text { EAB: } \mathrm{pH} \text { de } 7,22 ; \\
\mathrm{pO}_{2}: 34 \mathrm{mmHg} ; \mathrm{pCO}_{2}: 25 \mathrm{mmHg} ; \\
\mathrm{HCO}_{3}: 12,3 \mathrm{mmol}_{\mathrm{L}} ; \\
\text { EB: }-16,6 \mathrm{mmol} / \mathrm{L} ; \text { láctico: } 10,3 \mathrm{mmol} / \mathrm{L} \text {. } \\
\text { Hemograma (periférico): } \\
\text { noglobina de } 9,5 \mathrm{~g} / \mathrm{dl} \text {; hematocrito: el 29,8 } \\
\text { eticulocitos: el 6,8\%; el resto estaba normal, } \\
\text { incluidas las plaquetas. }\end{array}$ & $\begin{array}{l}\text { Exanguinotransfusión } \\
\text { isovolumétrica. } \\
\%\end{array}$ \\
\hline $\begin{array}{l}12 \text { h de vida } \\
\text { (postexang.) }\end{array}$ & $\begin{array}{c}\text { Mejoría del estado general, } \\
\text { más activo y vital. }\end{array}$ & $\begin{array}{l}\text { SatO del } 100 \% . \\
\text { TA: } 69 / 45 \mathrm{mmHg} . \\
\text { FC: } 140 \mathrm{lpm} .\end{array}$ & $\begin{array}{c}\text { EAB: } \mathrm{pH} \text { de } 7,41 ; \mathrm{pO}_{2}: 48 \mathrm{mmHg} \\
\mathrm{pCO}_{2}: 34 \mathrm{mmHg} ; \mathrm{HCO}_{3}: 22,6 \mathrm{mmol} / \mathrm{L} ; \\
\text { EB: }-3 \mathrm{mmol} / \mathrm{L} \text {; láctico: } 5,7 \mathrm{mmol} / \mathrm{L} \text {. } \\
\text { Hemograma: hemoglobina de } 13,5 \mathrm{~g} / \mathrm{dl} \text {; } \\
\text { hematocrito: el } 38,8 \% \text {; reticulocitos: } \\
\text { el } 5 \% \text {; plaquetas: } 33000 / \mathrm{mcl} ; \\
\text { leucocitos: } 3140 / \mathrm{mcl} .\end{array}$ & Se retira la $C P A P$. \\
\hline
\end{tabular}

UCIN: Unidad de Cuidados Intensivos Neonatales; $\mathrm{SatO}_{2}$ : saturación de oxígeno; TA: tensión arterial; FC: frecuencia cardíaca; lpm: latidos por minuto; EAB: estado ácido base; $\mathrm{pO}_{2}$ : presión parcial de oxígeno; $\mathrm{pCO}_{2}$ : presión parcial de dióxido de carbono; $\mathrm{HCO}_{3}$ : bicarbonato; CPAP: presión positiva continua en la vía aérea; TAM: tensión arterial media; EB: exceso de base; SSF: solución salina fisiológica. 
aumento de la hepatomegalia. Ante la sobrecarga de volumen, se realizó, a las 4 horas de vida, una exanguinotransfusión isovolumétrica, con buena evolución clínica. Se suspendió la $C P A P$, se normalizó la hemoglobina y presentó corrección de la acidosis a las 12 horas de vida. Secundarias a la exanguinotransfusión, se observaron plaquetopenia, leucopenia y neutropenia leves, que se normalizaron en las siguientes 72 horas (Tabla 1). El test de Kleihauer materno fue del 3,08 \%, por lo que se confirmó el diagnóstico de TFM masiva.

Ecografía transfontanelar (48 horas de vida): leves signos de edema cerebral. Buena evolución clínica y neurológica; las ecografías cerebrales seriadas mejoraron respecto a la primera, y se otorgó el alta a los 15 días de vida. El seguimiento neurológico presentó un retraso de enderezamiento a los 8 meses de edad, con mejoría progresiva tras la estimulación temprana. La exploración neurológica a los 24 meses fue normal.

\section{DISCUSIÓN}

La TFM es el paso de eritrocitos fetales a la circulación materna antes del parto o durante este. En la placenta, ocurre, fundamentalmente, al final del embarazo, un intercambio bidireccional de células y ácido desoxirribonucleico (ADN) fisiológico, sin significación clínica. Cuando el volumen es mayor de $30 \mathrm{ml}$ y existe incompatibilidad sanguínea, se produce la isoinmunización materna. La TFM se considera masiva con valores mayores de $80-150 \mathrm{ml}$ o más de $20 \mathrm{ml} / \mathrm{kg}$ de peso, aunque no hay consenso. Su frecuencia es desconocida; se estima en el 0,2-1\%o. $\mathrm{Si}$ es aguda, hay mayor riesgo de mortalidad. Si es crónica, de hidrops o secuelas neurológicas. ${ }^{1-3}$
La etiología es desconocida en más del $80 \%$. Se ha asociado a complicaciones obstétricas, traumatismos e hipertonía uterina (Tabla 2) ${ }^{1-4}$

En una revisión de 150 casos, se describieron los síntomas de presentación prenatal (Tabla 3). ${ }^{1,2}$ El signo clásico de disminución de movimientos fetales aparece en el $26 \%$. También se presentan como mortinatos (el 12,5\%) y RCTG alterado (en menos del $10 \%$, el patrón sinusoidal es patognomónico). Generalmente, la madre permanece asintomática, pero puede presentar cuadros similares a reacción transfusional (el $0,8 \%)$ y aumento súbito del hematocrito. ${ }^{1-4}$

La clínica es muy variada (Tabla 4). Se debe sospechar ante un neonato con anemia de causa desconocida, no isoinmune, con índice de reticulocitos elevado (más altos cuanto más crónica sea la instauración). La afectación clínica depende, entre otras, de la cantidad de sangrado y del tiempo de instauración.

TABLA 3. Signos y sintomas prenatales de presentación de hemorragia feto-materna mayor de $50 \mathrm{ml}$. Revisión de 150 casos. Adaptado de Blair J. Wylie ${ }^{1}$

\begin{tabular}{lc}
\hline & $\mathbf{N}(\%)$ \\
\hline Anemia prenatal & $42(35,0)$ \\
Disminución o ausencia de movimientos fetales & $32(26,7)$ \\
Muerte fetal & $15(12,5)$ \\
Hidrops fetal & $9(7,5)$ \\
Trazado fetal sospechoso (sinusoidal) & $8(6,7)$ \\
Restricción de crecimiento intrauterino & $4(3,3)$ \\
Trazo fetal sinusoidal & $2(1,7)$ \\
Fibrilación auricular fetal & $1(0,8)$ \\
Reacción transfusional materna & $1(0,8)$ \\
Incierto & $6(5,0)$ \\
\hline
\end{tabular}

TABLA 2. Factores de riesgo asociados a la transfusión feto-materna $a^{2,5}$

\begin{tabular}{|c|c|c|c|}
\hline Características maternas & Características fetales & $\begin{array}{c}\text { Características de la } \\
\text { placenta/útero }\end{array}$ & $\begin{array}{c}\text { Intervenciones/ } \\
\text { eventos }\end{array}$ \\
\hline Raza negra/etnia hispana & Anomalías fetales & Abruptio & Amniocentesis/ \\
\hline Estatus & Embarazo múltiple & Anomalías de la & procedimientos invasivos \\
\hline socioeconómico bajo & Pretérmino & vena umbilical (vasa previa) & Versión cefálica externa \\
\hline Región geográfica de parto & & Corioamnionitis & Traumatismo abdominal \\
\hline Preeclampsia & & Embarazo múltiple & (accidentes de tránsito con \\
\hline Seguimiento en una & & Tumores placentarios & cinturón, traumatismo \\
\hline clínica privada & & Hipertonía por oxitocina & abdominal, caídas) \\
\hline \multicolumn{4}{|l|}{ Mujer trabajadora en } \\
\hline horario nocturno & & & \\
\hline
\end{tabular}


El diagnóstico se basa en la detección de hematíes fetales en la sangre materna (test de Klehiauer o citometría de flujo). ${ }^{1,4,5}$ Este test es el método de referencia. Mide la hemoglobina $\mathrm{F}$ en la sangre materna y es positivo si es $>0,1 \%$. Actualmente, se puede usar la citometría de flujo, que mide el número de células fetales presentes en la sangre materna por medio de anticuerpos monoclonales específicos de la hemoglobina F. Ofrece ventajas: es automatizada (menos de 60 minutos), tiene mayor objetividad y precisión, y es más rápida. No se puede realizar de forma urgente, debido a que la mayoría de los hospitales no cuentan con la técnica. ${ }^{1,5}$ Ambas pruebas se pueden realizar a la madre antes del parto o en el nacimiento lo más precozmente posible. El volumen de sangre fetal observado en la circulación materna es predictor de gravedad. ${ }^{1}$

En nuestro caso, el test de Klehiauer era positivo: 3,08\%. Para el manejo, es importante estimar el volumen fetal perdido. Se usan distintas fórmulas; la más empleada es la fórmula de Creasy: porcentaje de células fetales en la sangre materna x 5000/100. En este caso, un test del 3,08 \% equivale a $152 \mathrm{ml}$ aproximadamente o $65 \mathrm{cc} / \mathrm{kg}$ de volemia del recién nacido para su peso.

Si el diagnóstico es posnatal, como en el presente paciente, el tratamiento consiste en revertir esa anemia grave y tratar las complicaciones. ${ }^{4-8}$ En caso de anemia aguda, es preciso restaurar la volemia y la capacidad de transporte de $\mathrm{O}_{2}$ de forma urgente (inicialmente, con suero salino fisiológico, de Ringer o sangre de grupo $0 \mathrm{Rh}$ negativo si es necesario) para evitar la isquemia propia de la hipovolemia. En caso de anemia crónica, el feto ha activado intraútero los mecanismos de compensación. Al nacer, está normovolémico, pero su capacidad de transporte de oxígeno está disminuida, por lo que el objetivo es aumentar la hemoglobina y evitar la sobrecarga de volumen. Alcanzar el equilibrio entre los dos es fundamental para que el tratamiento de esta patología sea óptimo. ${ }^{4-8}$ Por ello, en caso de anemia grave crónica con repercusión hemodinámica que empeora o no mejora tras una primera transfusión, algunos autores recomiendan la realización de una exanguinotransfusión isovolumétrica con recambio simple de volemia $(80-100 \mathrm{ml} / \mathrm{kg}){ }^{4,10}$ Ante un recién nacido con anemia de causa desconocida y sospecha de TFM, se recomienda iniciar la expansión con volumen y monitorizar la respuesta (relleno capilar, hepatomegalia, medición de la presión venosa central, ecografía funcional, equilibrio ácido base, etc.). ${ }^{5-7}$

En nuestro caso, se inició la expansión, la transfusión de sangre y la administración de diuréticos con mala tolerancia hemodinámica; presentó signos de sobrecarga de volumen y persistencia de acidosis láctica a pesar de la mejoría de los niveles de hemoglobina. En las primeras horas de vida, se realizó la exanguinotransfusión, con una clara mejoría hemodinámica y respiratoria, y la normalización de los parámetros a las 12 horas.

Llaman la atención varios artículos publicados de casos clínicos que presentan una anemia con hemoglobina $<5 \mathrm{~g} / \mathrm{dl}$ y que indican como único tratamiento transfusión y diuréticos, algunos de ellos, con evolución fatal para el paciente por insuficiencia cardíaca tras múltiples transfusiones de concentrado de hematíes, sin mencionarse la posible indicación de exanguinotransfusión. En estos estudios, la tasa de mortalidad y de secuelas neurológicas es elevada. ${ }^{3,7,11}$ No hay estudios a largo plazo de evolución neurológica de recién nacidos que hayan precisado tratamiento con exanguinotransfusión.

TABLA 4. Signos y síntomas de presentación posnatales de hemorragia feto-materna. Adaptado de Markham, Lori A. ${ }^{5}$

Signos y síntomas de presentación posnatales de hemorragia feto-materna

Anemia neonatal de causa no conocida
Shock hipovolémico
Hipotensión
Hidrops fetal
Asfixia
Síndrome de dificultad respiratoria
Hipertensión pulmonar persistente
Encefalopatía hipóxico-isquémica
Falla multiorgánica (miocardiopatía, falla renal aguda, coagulación intravascular diseminada, enterocolitis necrotizante)
Parálisis cerebral
Muerte fetal intraútero o al nacer 
En el tratamiento de la TFM, debe tenerse en cuenta la gravedad de la anemia y el tiempo de instauración. En la anemia crónica, se deberían vigilar los signos de sobrecarga de líquidos y, si aparecieran, se podría plantear realizar una exanguinotransfusión. Son necesarios estudios bien diseñados, con mayor número de casos para establecer las indicaciones de cada tratamiento y evaluar la evolución a largo plazo.

\section{REFERENCIAS}

1. Wylie BJ, D'Alton ME. Fetomaternal hemorrhage. Obstet Gynecol. 2010; 115(5):1039-51.

2. Stroustrup A, Plafkin C, Tran T, Savitz DA. Demographic and Behavioral Predictors of Severe Fetomaternal Hemorrhage: a Case-Control Study. Neonatology. 2016; 109(4):248-54.

3. Stroustrup A, Trasande L. Demographics, clinical characteristics and outcomes of neonates diagnosed with fetomaternal haemorrhage. Arch Dis Child Fetal Neonatal Ed. 2012; 97(6):F405-10.
4. Stefanovic V. Fetomaternal hemorrhage complicated pregnancy: Risks, identification, and management. Curr Opin Obstet Gynecol. 2016; 28(2):86-94.

5. Markham LA, Charsha DS, Perelmuter B. Case Report of Massive Fetomaternal Hemorrhage and a Guideline for Acute Neonatal Management. Adv Neonatal Care. 2006; 6(4):197-205.

6. De Luca D, Pietrini D, Piastra M, Tiberi E, et al. Successful resuscitation of unexpected neonatal hemorrhagic shock due to massive feto-maternal hemorrhage. Paediatr Anaesth. 2008; 18(10):1004-6.

7. Solomonia N, Playforth K, Reynolds E. Fetal-Maternal Hemorrhage: A Case and literature Review. AJP Rep. 2012; 2(1):7-14.

8. Naulaers G, Barten S, Vanhole C, Verhaeghe J, et al. Management of severe neonatal anemia due to fetomaternal transfusion. Am J Perinatol. 1999; 16(4):193-6.

9. Murray NA, Roberts IA. Neonatal transfusion practice. Arch Dis Child Fetal Neonatal Ed. 2004; 89(2): F101-7.

10. Girelli G, Antoncecchi S, Casadei AM, Del Vecchio A, et al. Recommendations for transfusion therapy in neonatology. Blood Transfus. 2015; 3(3):484-97.

11. Kadooka M, Kato H, Kato A, Ibara S, et al. Effect of neonatal hemoglobin concentration on long-term outcome of infants affected by fetomaternal hemorrhage. Early Hum Dev. 2014; 90(9):431-4 